\title{
Obésité et affections liées à l'obésité dans une population des Premières nations du Canada*
}

\author{
S.G. Bruce, Ph. D. (1); N.D. Riediger, M. Sc. (1); J.M. Zacharias, M.D., M. Sc. (1); T.K. Young, M.D., Ph. D. (2)
}

${ }^{*}$ Cet article fait l'objet d'une publication conjointe par Preventing Chronic Disease et Maladies chroniques au Canada. Preventing Chronic Disease en est le premier éditeur, Maladies chroniques au Canada le second. La publication originale est : Bruce SG, Riediger ND, Zacharias JM, Young TK. Obesity and obesity-related comorbidities in a Canadian First Nation population. Prev Chronic Dis 2011;8(1). Consultable en ligne à la page : http://www.cdc.gov/pcd/issues/2011/jan/09_0212.htm

\section{Résumé}

Introduction : Au Canada, on observe des taux d'obésité plus élevés au sein des populations autochtones des Premières nations qu'au sein des autres populations. Nous avons étudié l'obésité et les affections liées à l'obésité dans une communauté des Premières nations du Manitoba.

Méthodologie : Nous avons mené, en 2003, une étude de dépistage du diabète et des complications du diabète qui nous a permis de sélectionner un échantillon représentatif d'adultes des Premières nations du Manitoba $(\mathrm{N}=483)$. Nous avons évalué les affections chroniques et les facteurs de risque d'affections chroniques.

Résultats : La prévalence de l'obésité et des affections liées à l'obésité était plus élevée chez les femmes que chez les hommes. À l'aide d'une analyse multivariée, nous avons établi que les facteurs étroitement liés à l'obésité chez les femmes étaient la tension artérielle diastolique, l'insulinorésistance et la situation d'emploi. Chez les hommes, les facteurs relevés étaient l'âge, les taux d'apolipoprotéine A1 et d'apolipoprotéine B et l'insulinorésistance. Soixante-quinze pour cent des participants à l'étude présentaient au moins un des problèmes de santé suivants : obésité, dyslipidémie, hypertension ou diabète. La comorbidité était élevée même dans les groupes d’âge les plus jeunes : 22 \% des hommes et $43 \%$ des femmes de 18 à 29 ans présentaient au moins deux affections chroniques. Vingt-deux pour cent des participants étaient atteints d'une hypertension non diagnostiquée. Ces derniers présentaient un nombre sensiblement plus élevé d'affections chroniques et risquaient davantage d'être atteints de microalbuminurie que les sujets sans hypertension. Il n'y avait pas de différence significative quant au nombre d'affections chroniques entre les participants dont le diagnostic d'hypertension venait d'être posé et ceux dont le diagnostic avait déjà été établi.

Conclusions : La prévalence de l'obésité et d'autres affections chroniques est élevée dans la communauté étudiée, en particulier chez les jeunes. Des interventions communautaires sont actuellement menées dans le but de réduire le taux excessif de problèmes de santé.

Mots clés : cardiovasculaire, santé communautaire, diabète, minorité, obésité, dépistage, diabète de type 2, Premières nations, Indiens d'Amérique du Nord

\section{Introduction}

La population des Premières nations du Canada est en moins bonne santé que la population canadienne en général ${ }^{1}$, notamment au chapitre des maladies chroniques, des facteurs de risque de maladie chronique ${ }^{2}$ ainsi que des blessures et des accidents ${ }^{3}$. Au Canada, les membres des Premières nations constituent un des trois groupes autochtones reconnus par la Constitution, les deux autres étant les Métis et les Inuits. Dans le cadre du présent article, le terme " autochtone » a été utilisé lorsque la recherche portait sur au moins deux de ces groupes et qu'il n'y avait aucune distinction entre les groupes lors de l'analyse. Toutefois, si la recherche comprenait un seul groupe, nous avons identifié ce dernier. D’après les données de l'Enquête sur la santé dans les collectivités canadiennes de 2005-2006, la prévalence de l'obésité chez les personnes qui se sont déclarées autochtones et qui ne vivaient pas dans des réserves atteignait $20 \%$ dans le Nord du Canada (Yukon, Territoires du Nord-Ouest et Nunavut) et $23 \%$ dans le reste du Canada ${ }^{4}$.

La prévalence de l'obésité semble être plus élevée chez les membres des Premières nations qui habitent dans les réserves. À Sandy Lake, en Ontario, la prévalence de l'obésité (indice de masse corporelle $[\mathrm{IMC}] \geq 30 \mathrm{~kg} / \mathrm{m}^{2}$ ) s'établissait à $50 \%$ chez les hommes et à $65 \%$ chez les femmes ${ }^{5}$. De plus, dans une communauté des Premières nations du Québec, $91 \%$ des sujets (parmi un échantillon de 172 personnes) présentaient une obésité abdominale ${ }^{6}$. La prévalence de l'obésité se situait à $55 \%$ dans un échantillon de membres des Premières nations de l'Alberta et à $49 \%$ dans un échantillon de Métis?.

La prévalence des affections liées à l'obésité est également élevée chez les membres

Rattachement

1. Université du Manitoba, Winnipeg (Manitoba), Canada

2. Université de Toronto, Toronto (Ontario), Canada

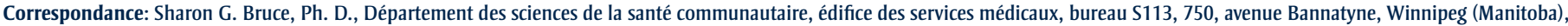
Canada R3E 0W3; tél. : 204-975-7745; courriel : brucesg@cc.umanitoba.ca. 
des Premières nations du Canada. La prévalence du diabète dans ces populations est de 3 à 5 fois supérieure à celle qu'on retrouve dans la population canadienne en général ${ }^{5-8}$. L'hypertension, la dyslipidémie, le syndrome métabolique et les complications du diabète telles que les maladies cardiovasculaires (MCV), les accidents vasculaires cérébraux (AVC), la rétinopathie, la neuropathie et la néphropathie contribuent aussi de façon importante au piètre état de santé observée,5-10. Les MCV constituent la principale cause de mortalité au Canada, et les populations autochtones présentent un taux de mortalité par $\mathrm{MCV}$ deux fois plus élevé que les populations non autochtones ${ }^{2}$. Dans un échantillon aléatoire, le taux de MCV se situait à $18 \%$ chez les Autochtones et à $8 \%$ chez les Canadiens d'ascendance européenne ${ }^{2}$.

Même si les données indiquent une prévalence excessive d'obésité, de diabète et de troubles métaboliques associés dans les populations des Premières nations du Canada, peu de chercheurs se sont penchés sur leur coexistence au sein de cette population. Nous avions pour objectif d'explorer l'ampleur et l'effet de l'obésité et des affections liées à l'obésité dans une Première nation du Manitoba.

\section{Méthodologie}

Notre méthodologie a déjà été décrite ${ }^{10}$. En bref, 483 résidents admissibles d'une communauté des Premières nations du Manitoba se sont portés volontaires, en 2003, pour participer à une étude de dépistage du diabète et des complications du diabète. Les 1356 participants admissibles étaient des personnes adultes, non enceintes, âgées de 18 ans et plus, inscrites comme Indien et résidant dans la communauté. Notre échantillon (36\%, 483 personnes sur 1 356) est représentatif des participants admissibles en fonction de l'âge et du sexe ${ }^{10}$. Une infirmière a prélevé des échantillons de sang veineux afin de mesurer les taux de glucose, d'hémoglobine A1c, d'insuline, de cholestérol total, de cholestérol HDL (lipoprotéines de haute densité), de triglycérides, d'apolipoprotéines A1 (apoA1), d'apolipoprotéines $\mathrm{B}(\mathrm{apoB})$ totales et d'homocystéine des participants à jeun. Le cholestérol LDL (lipoprotéines de faible densité) a été calculé.
Une infirmière ou un assistant de recherche qualifié a fait répondre les participants à un questionnaire en 17 points portant notamment sur les données de population habituelles (âge, sexe, situation d'emploi, niveau de scolarité), les habitudes de tabagisme passées et actuelles, le nombre de cigarettes fumées par jour, les diagnostics antérieurs de diabète et d'hypertension (" Est-ce qu'un médecin vous a déjà dit que vous aviez le diabète? ", " Depuis quand êtes-vous atteint du diabète? ») et la médication actuelle. Les mesures anthropométriques ont été prises à l'aide des techniques courantes ${ }^{11}$. On a mesuré la taille à l'aide d'un ruban métrique fixé au mur et d'une équerre (résultat arrondi au 0,5 cm le plus proche), le poids, au moyen d'une balance (résultat arrondi au $0,1 \mathrm{~kg}$ le plus proche), le tour de taille, au point le plus étroit ou à la hauteur de la douzième côte (résultat arrondi au $0,5 \mathrm{~cm}$ le plus proche) et le tour de hanches, au niveau de la symphyse pubienne et du point le plus large des fesses (résultat arrondi au 0,5 $\mathrm{cm}$ le plus proche) ${ }^{11}$.

Dans le cadre de notre étude, nous avons défini l'obésité abdominale par un tour de taille supérieur à $102 \mathrm{~cm}$ chez les hommes et à $88 \mathrm{~cm}$ chez les femmes ${ }^{10}$; le diabète, par un taux de glucose plasmatique à jeun d'au moins 7,0 mmol/L ou un diagnostic antérieur; l'hyperglycémie modérée à jeun, par un taux de glucose plasmatique à jeun de 6,1 à 6,9 mmol/L $\mathrm{L}^{12}$; l'hypertension, par une tension artérielle systolique supérieure à $140 \mathrm{~mm} \mathrm{Hg}$, une tension artérielle diastolique supérieure à $90 \mathrm{~mm} \mathrm{Hg}$ ou un diagnostic antérieur et la dyslipidémie, par un taux de triglycérides plasmatiques d'au moins $1,7 \mathrm{mmol} / \mathrm{L}$ et un taux de cholestérol HDL d'au plus 1,03 mmol/L chez les hommes et d'au plus $1,30 \mathrm{mmol} / \mathrm{L}$ chez les femmes. Par ailleurs, la définition du syndrome métabolique répondait aux critères de l'Adult Treatment Panel III'13; l'insulinorésistance, à l'estimation fondée sur le modèle d'évaluation homéostatique (HOMA), calculée comme suit : [(insuline [pmol] x 0,139) x (glucose $[\mathrm{mmol} / \mathrm{L}] / 22,5)]$; la microalbuminurie, à un ratio albumine/créatinine supérieur à 2,0 $\mathrm{mg} / \mathrm{mmol}$ chez les hommes et supérieur à 2,8 mg/mmol chez les femmes et la neuropathie, à la présence d'engourdissements, de picotements et de douleur et à la perte de sensation protectrice déterminée par l'application, par une infirmière ayant d'abord effectué l'examen du pied, du monofilament de $10 \mathrm{~g}$ de SemmesWeinstein (Sensory Testing Systems, Baton Rouge, Louisiane) ${ }^{14}$. Le Comité d'éthique de la recherche en santé de l'Université du Manitoba a approuvé le projet.

Les analyses statistiques ont été effectuées à l'aide de la version 16 de SPSS pour Windows (IBM, Chicago, Illinois). Nous avons utilisé des tests $\chi^{2}$ afin de détecter les différences entre les sexes en ce qui a trait à la prévalence des affections chroniques, aux facteurs de risque et aux variables sociodémographiques. Nous avons comparé les différences entre les sexes à l'aide de tests $t$, dans le cas des variables à distribution continue, et à l'aide de tests de Mann-Whitney, dans le cas des variables à distribution non normale. Les différences relatives au nombre d'affections chroniques selon le groupe d'âge et le sexe et les différences relatives au nombre d'affections concomitantes selon la situation relative à l'hypertension ont été déterminées à l'aide de tests $\chi^{2}$ bilatéraux. Les différences étaient considérées significatives lorsque $\mathrm{p}<0,05$. Nous avons utilisé la régression logistique afin d'estimer les rapports de cotes (RC) pour l'obésité et la microalbuminurie, avec des intervalles de confiance (IC) de $95 \%$. Les participants pour lesquels des données étaient manquantes ont été exclus des analyses. Aucun profil n'a été observé relativement aux valeurs manquantes selon le sexe, le groupe d'âge, la présence d'affections chroniques ou les facteurs de risque.

\section{Résultats}

Les caractéristiques démographiques et sanitaires des sujets de l'étude font état d'une population jeune, peu instruite et confrontée à un taux de chômage élevé (tableau 1). La prévalence du tabagisme, du diabète, de l'hypertension, du surpoids et de l'obésité était élevée parmi les sujets. Les données relatives au tour de taille de 259 participants obèses (sur 264) étaient disponibles; pour 96 \% d'entre eux (250 sur 259), ces données correspondaient à un risque élevé de problèmes de santé 9 . Nous n’avons noté aucune différence 
TABLEAU 1

Caractéristiques de la population des Premières nations $(\mathrm{N}=483)$, Manitoba, Canada, 2003

\begin{tabular}{|c|c|}
\hline Caractéristique $^{\mathrm{a}}$ & Nombre (poucentage) \\
\hline \multicolumn{2}{|l|}{ Sexe, n (\%) } \\
\hline Hommes & $230(48)$ \\
\hline Femmes & $253(52)$ \\
\hline Âge (ans), moyenne (ET) & $37,8(12,3)$ \\
\hline \multicolumn{2}{|l|}{ Scolarité (n = 469), n (\%) } \\
\hline Neuvième année ou plus & $220(47)$ \\
\hline Moins d'une neuvième année & $249(53)$ \\
\hline \multicolumn{2}{|l|}{ Situation d'emploi ( $n=476), n(\%)$} \\
\hline Avec emploi & $137(29)$ \\
\hline Sans emploi & $339(71)$ \\
\hline \multicolumn{2}{|l|}{ A déjà fumé ( $n=477), n(\%)$} \\
\hline Oui & $391(82)$ \\
\hline Non & $86(18)$ \\
\hline \multicolumn{2}{|l|}{ Fumeur $(n=471), n(\%)$} \\
\hline Oui & $349(74)$ \\
\hline Non & $122(26)$ \\
\hline \multicolumn{2}{|l|}{ IMC, $\mathrm{kg} / \mathrm{m}^{2}(\mathrm{n}=468), \mathrm{n}(\%)$} \\
\hline$<25,0$ & $76(16)$ \\
\hline $25,0-29,9$ & $128(27)$ \\
\hline$\geq 30,0$ & $264(56)$ \\
\hline Syndrome métabolique $^{\mathrm{b}}(\mathrm{n}=475), \mathrm{n}(\%)$ & $252(53)$ \\
\hline Obésité abdominale $\quad(n=464)$ & $313(68)$ \\
\hline Diabète $^{\mathrm{d}}(\mathrm{n}=483), \mathrm{n}(\%)$ & $140(29)$ \\
\hline Hypertension $^{\mathrm{e}}(\mathrm{n}=472), \mathrm{n}(\%)$ & $201(43)$ \\
\hline Dyslipidémie $^{f}(\mathrm{n}=483), \mathrm{n}(\%)$ & $155(32)$ \\
\hline Microalbuminurie $^{8}(n=466), n(\%)$ & $94(20)$ \\
\hline
\end{tabular}

Abréviations : ET, écart type; IMC, indice de masse corporelle.

a Les numérateurs se situent entre 464 et 483 , car certains sujets n'ont pas suivi le protocole au complet.

${ }^{\mathrm{b}}$ D'après les critères de l'Adult Treatment Panel III' ${ }^{13}$.

' Correspond à une valeur $>102 \mathrm{~cm}$ chez les hommes et $>88 \mathrm{~cm}$ chez les femmes.

${ }^{d}$ Correspond à un diagnostic antérieur ou à une glycémie à jeun $\geq 7,0 \mathrm{mmol} / \mathrm{L}$.

${ }^{\mathrm{e}}$ Correspond à une tension artérielle systolique $>140 \mathrm{~mm} \mathrm{Hg}$ ou à une tension artérielle diastolique $>90 \mathrm{~mm} \mathrm{Hg}$ ou à un diagnostic antérieur.

${ }^{\mathrm{f}}$ Correspond à un taux de triglycérides plasmatiques $\geq 1,7 \mathrm{mmol} / \mathrm{L}$ et à un taux de cholestérol $\mathrm{HDL} \leq 1,03 \mathrm{mmol} / \mathrm{L}$ chez les hommes et $\leq 1,30 \mathrm{mmol} / \mathrm{L}$ chez les femmes.

\section{FIGURE 1}

Prévalence de l'obésité selon l'âge et le sexe dans une population des Premières nations du Canada.

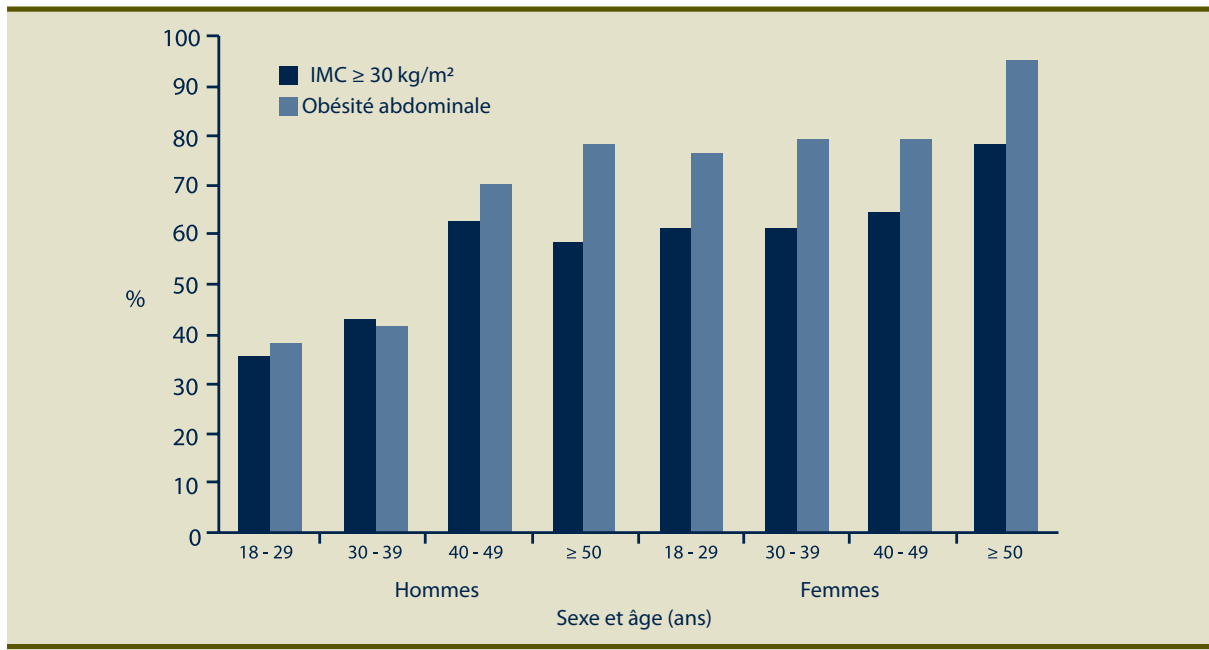

IMC : indice de masse corporelle.

Abréviations : L'obésité abdominale correspond à un tour de taille supérieur à $102 \mathrm{~cm}$ chez les hommes et à $88 \mathrm{~cm}$ chez les femmes. [Ces données sont également disponibles sous forme de tableau.] importante entre les hommes et les femmes en ce qui concerne la prévalence du diabète et de l'hypertension. Cependant, la prévalence de la dyslipidémie était beaucoup plus élevée chez les femmes (38\%) que chez les hommes (26\%).

\section{Obésité globale et obésité abdominale}

Nous avons réparti les participants obèses selon l'âge et le sexe, et en fonction de la mesure de leur IMC et de leur tour de taille (figure 1). Près de $50 \%$ des hommes et de $65 \%$ des femmes étaient obèses (selon leur IMC) et $53 \%$ des hommes ainsi que $81 \%$ des femmes présentaient une obésité abdominale. L'obésité était plus fréquente chez les femmes que chez les hommes selon l'IMC $\left(\chi^{2}=14,62 ; p<0,001\right)$ et selon l'obésité abdominale $\left(\chi^{2}=41,38 ; p<0,001\right)$. La prévalence de l'IMC $\geq 30 \mathrm{~kg} / \mathrm{m}^{2}$ était plus élevée chez les femmes de 18 à 29 ans que chez les hommes du même groupe d'âge $\left(\chi^{2}=\right.$ 9,06; $\left.p<0,01\right)$. L'obésité abdominale était beaucoup plus fréquente chez les femmes que chez les hommes pour tous les groupes d'âge, sauf celui des 40 à 49 ans. Les trois-quarts des femmes de 18 à 29 ans présentaient une obésité abdominale.

Compte tenu des différences observées entre les hommes et les femmes relativement à l'obésité et de la grande prévalence de l'obésité abdominale, nous avons déterminé quels étaient les facteurs associés à l'obésité abdominale pour chaque sexe à l'aide d'une analyse multivariée par régression logistique descendante pas à pas. Les variables comprises dans les modèles étaient celles étroitement associées à l'obésité abdominale dans les analyses bivariées. Chez les femmes, ces variables étaient l'âge, la tension artérielle systolique et diastolique, les taux de triglycérides, d'apoA1 et d'apoB, l'insulinorésistance, le niveau de scolarité et la situation d'emploi. Chez les hommes, les variables comprises dans le modèle étaient l'âge, la tension artérielle systolique et diastolique, les taux de triglycérides, d'apoA1 et d'apoB, l'insulinorésistance et la microalbuminurie (tableau 2).

Chez les femmes, le risque d'être atteinte d'obésité abdominale augmentait avec la tension artérielle diastolique et l'insulinorésistance. De plus, le risque d'obésité était inférieur chez les femmes occupant 
TABLEAU 2

Risque d'obésité abdominale selon le sexe dans une population des Premières nations $(\mathrm{N}=483)$, Manitoba, Canada, 2003

\begin{tabular}{rlrrrrr}
\hline Sexe & \multicolumn{1}{c}{ Facteur de risque } & \multicolumn{2}{c}{$\beta$ (Er-T) } & \multicolumn{2}{c}{ RC (IC à 95 \%) } & Valeur $\boldsymbol{p}^{\mathrm{a}}$ \\
\hline \multirow{3}{*}{ Femme } & En emploi & $-1,16$ & $(0,45)$ & 0,31 & $(0,13-0,76)$ & 0,01 \\
& Tension artérielle & 0,05 & $(0,02)$ & 1,05 & $(1,01-1,10)$ & 0,03 \\
& diastolique & 1,14 & $(0,205)$ & 0,31 & $(0,13-0,76)$ & 0,01 \\
\hline \multirow{3}{*}{ Homme } & Insulinorésistance & 0,05 & $(0,01)$ & 1,05 & $(1,02-1,08)$ & 0,001 \\
& Âge & $-3,06$ & $(1,20)$ & 0,05 & $(0-0,49)$ & 0,01 \\
& ApoA1 & 1,54 & $(0,68)$ & 4,64 & $(1,22-17,65)$ & 0,02 \\
& ApoB & 0,33 & $(0,08)$ & 1,40 & $(1,19-1,63)$ & $<0,001$ \\
\hline
\end{tabular}

Abréviations : Er-T, erreur-type; RC, rapport de cotes; IC, intervalle de confiance; apo, apolipoprotéine.

${ }^{a}$ Calculée par régression logistique.

FIGURE 2

Pourcentage des sujets atteints d'affections chroniques (obésité, diabète, hypertension, dyslipidémie) selon l'âge et le sexe dans une population de Premières nations du Canada.

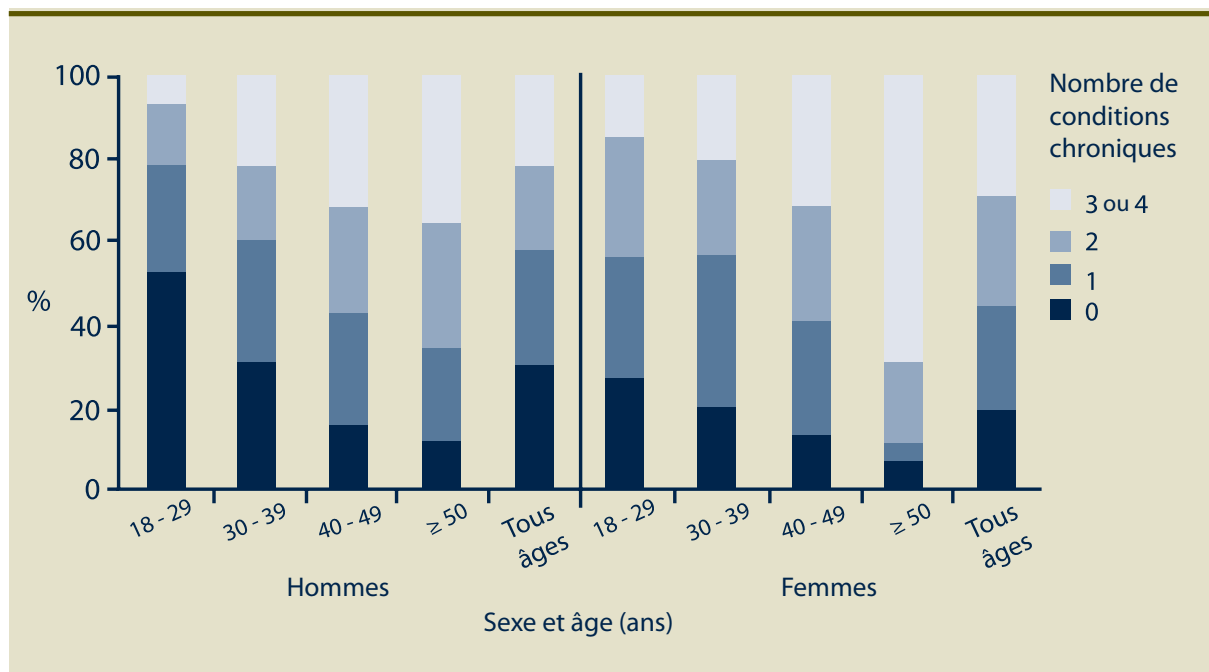

[Ces données sont également disponibles sous forme de tableau.]

un emploi. Chez les hommes, l'obésité abdominale était associée au vieillissement, à l'insulinorésistance, à un faible taux d'apoA1 et à un taux élevé d'apoB.

\section{Affections concomitantes}

Nous avons établi l'ampleur de la comorbidité au sein de cette population relativement à quatre affections chroniques : l'obésité, le diabète, l'hypertension et la dyslipidémie. La répartition des affections chroniques selon l'âge et le sexe (figure 2) a fait ressortir que les femmes de 18 à 29 ans et de 50 ans et plus étaient beaucoup plus touchées par les affections chroniques que les hommes des mêmes groupes d'âge. Vingt-deux pour cent des hommes (16 sur 73 ) et $43 \%$ des femmes (30 sur 69) âgés de 18 à 29 ans étaient atteints d'au moins deux affections chroniques évitables. Parmi les sujets qui présentaient une obésité abdominale, $48 \%$ (147 sur 303) étaient atteints d'hypertension et $35 \%$ (111 sur 313) souffraient de diabète. De ce nombre, $37 \%$ des sujets hypertendus (54 sur 147) et $26 \%$ des sujets diabétiques (29 sur 111) n’avaient pas été diagnostiqués.

Au total, $22 \%$ des participants à l'étude (72 sur 337) présentaient une hypertension non diagnostiquée. Nous avons comparé l'ampleur de la comorbidité chez les sujets dont l'hypertension venait d'être diagnostiquée à celle de deux groupes : 1) les sujets n'étant pas atteints d'hypertension et 2) les sujets dont l'hypertension avait déjà été diagnostiquée (tableau 3). Les sujets dont l'hypertension venait

\section{Hypertension non diagnostiquée}

d'être diagnostiquée étaient sensiblement plus nombreux à souffrir d'un plus grand nombre d'affections chroniques que les sujets affichant une tension artérielle normale. Toutefois, en ce qui concerne la comorbidité, nous n'avons pas observé de différence appréciable entre les sujets dont le diagnostic d'hypertension était récent et ceux dont le diagnostic avait déjà été établi. Pour ce qui est des résultats, le rapport de cotes ajusté relatif à la microalbuminurie chez les sujets venant de recevoir un diagnostic d'hypertension était presque deux fois plus élevé que chez les sujets sans hypertension. Le rapport de cotes ajusté relatif à la microalbuminurie chez les sujets dont le diagnostic d'hypertension avait déjà été établi était presque cinq fois plus élevé que chez les sujets sans hypertension $[p<0,001$ dans le tableau].

\section{Analyse}

La prévalence de l'obésité dans la population étudiée est l'une des plus élevées ayant été signalées pour une communauté des Premières nations du Canada dont les membres vivent dans une réserve $e^{6,7}$. Cette prévalence est beaucoup plus élevée que celle observée dans la population canadienne en général ${ }^{4}$ et dans les populations autochtones ne vivant pas dans des réserves ${ }^{4,15}$. La forte prévalence de l'obésité dans la population étudiée est préoccupante étant donné le rôle déterminant de l'obésité dans l'apparition du diabète, des maladies cardiaques, des AVC et de certains cancers. Par ailleurs, la prévalence du diabète que nous avons observée figure parmi les plus élevées ayant été signalées parmi les populations des Premières nations du Canada ${ }^{6,7,16}$.

Une autre constatation préoccupante est la forte prévalence de l'obésité chez les jeunes adultes, en particulier chez les jeunes femmes en âge de procréer. Les relations entre l'obésité maternelle et le diabète gestationnel, le diabète de type 2 , les mauvaises conditions à la naissance ainsi que l'apparition de l'obésité et du diabète de type 2 chez les enfants ont déjà été bien établies ${ }^{17-20}$. Par conséquent, la prévalence de l’obésité au sein de cette population justifie une intervention. Ces 
TABLEAU 3

Affections concomitantes et risque de microalbuminurie selon la situation relative à l'hypertension dans une population des Premières nations, Manitoba, Canada, 2003

\begin{tabular}{|c|c|c|c|c|c|c|c|c|}
\hline \multirow{3}{*}{$\begin{array}{l}\text { Situation relative à l'hyperten- } \\
\text { sion chez les participants } \\
(\mathbf{N}=453)^{\mathrm{b}}\end{array}$} & \multicolumn{5}{|c|}{$\mathrm{N}^{\text {bre }}$ de participants (\%) } & \multicolumn{3}{|c|}{ Risque de microalbuminurie $^{a}$} \\
\hline & \multicolumn{4}{|c|}{$\mathbf{N}^{\text {bre }}$ d'affections concomitantes } & \multirow{2}{*}{ Valeur $\boldsymbol{p}^{c}$} & \multirow{2}{*}{$\beta(\mathrm{Er}-\mathrm{T})$} & \multirow{2}{*}{$\mathbf{R C}$} & \multirow{2}{*}{ Valeur $\boldsymbol{p}^{c}$} \\
\hline & 0 & 1 & 2 & 3 & & & & \\
\hline Sans hypertension $(n=263), n(\%)$ & $111(42)$ & $88(33)$ & $50(19)$ & $14(5)$ & 1 [Référence] & 1 [Référence] & 1,000 & 1 [Référence] \\
\hline Diagnostic récent d'hypertension ( $n=72), n(\%)$ & $17(24)$ & $20(28)$ & $19(26)$ & $16(22)$ & $<0,001$ & $0,653(0,48-0,82)$ & 1,921 & $<0,001$ \\
\hline Diagnostic antérieur d'hypertension ( $n=118), n(\%)$ & $18(15)$ & $36(31)$ & $38(32)$ & $26(22)$ & 0,510 & $1,542(1,22-1,86)$ & 4,673 & $<0,001$ \\
\hline
\end{tabular}

Abréviations : Er-T, erreur-type; RC, rapport de cotes.

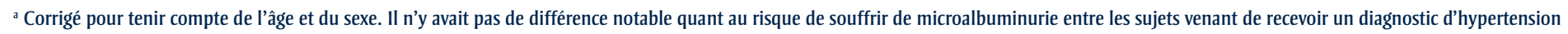
et ceux dont le diagnostic avait été établi auparavant.

${ }^{\mathrm{b}}$ Dans cette analyse, nous n'avons inclus que les participants dont les valeurs étaient disponibles pour toutes les variables.

'Calculée à l'aide du test $\chi^{2}$.

résultats sont importants pour deux raisons : 1) les sujets étaient atteints de problèmes de santé chroniques depuis leur jeune âge et 2) les cas d'hypertension et de diabète n'avaient pas été diagnostiqués chez une grande partie des sujets obèses.

Les résultats de l'analyse de régression logistique ont confirmé les associations déjà établies entre l'obésité et les taux de lipides plasmatiques, l'hypertension, l'insulinorésistance et les caractéristiques sociodémographiques de la population étudiée. Les lipides n'étaient pas prédicteurs de l'obésité abdominale chez les femmes dans les analyses de régression selon le sexe. À notre avis, deux raisons peuvent expliquer cet état de fait. La première : la prévalence de l'obésité abdominale était élevée chez les femmes de tous les groupes d'âge, mais celle de taux anormaux de lipides était faible. Les différences liées à l'âge pourraient avoir été atténuées par le fait que notre résultat (l'obésité) était présent dans tous les groupes d'âge. La seconde : des études antérieures ont montré des différences appréciables entre les sexes en ce qui a trait à la relation entre l'adiposité et les lipides plasmatiques $^{21}$. Puisque nous n'avons pas observé de taux anormaux de lipides chez les femmes, cette conclusion mérite un examen plus approfondi.

Nous avons remarqué une forte prévalence de la comorbidité même dans les groupes d'âges les plus jeunes. L'étude DRUID (Diabetes and Related conditions in Urban Indigenous people in the Darwin region) avait également fait ressortir que le nombre d'affections cardiovasculaires concomitantes chez les Aborigènes d'Australie était élevé et qu'il augmentait avec l'âge ${ }^{22}$. Une grande proportion des sujets de notre étude présentait un diabète ou une hypertension non diagnostiqués, malgré les fortes corrélations connues entre l'obésité, le diabète, la dyslipidémie et l'hypertension ${ }^{23}$ (il ne nous a pas été possible de déterminer le nombre de cas de dyslipidémie non diagnostiqués, car nous n'avons pas demandé aux sujets de déclarer s'ils avaient des taux anormaux de lipides). Une étude précédente a établi que les facteurs de risque associés à l'absence de prise de la tension artérielle étaient les suivants : le fait d'être un homme, de ne s'être jamais marié, de ne pas avoir de médecin régulier, d'être jeune et d'être un Autochtone ou un membre d'une minorité ethnique ${ }^{24}$. Dans notre étude, la probabilité d'être atteint d'une hypertension non diagnostiquée était plus élevée pour les hommes (RC, $3,27$; IC à $95 \%, 1,74-6,10 ; p<0,01)$ et pour les participants plus jeunes (RC, 1,04 ; IC à $95 \%, 1,01-1,07 ; p<0,001)$.

De plus, dans notre étude, l'hypertension non diagnostiquée n'était pas sans conséquence. L’ampleur de la comorbidité parmi les participants chez qui on venait de diagnostiquer une hypertension était semblable à celle observée chez les sujets dont le diagnostic avait déjà été établi. De plus, le risque d'être atteint de microalbuminurie était beaucoup plus élevé chez les sujets dont le diagnostic d'hypertension était récent que chez ceux ne souffrant pas d'hypertension, mais il était relativement semblable à celui des sujets dont le diagnostic avait déjà été établi. Cela nous porte à croire que les sujets nouvellement diagnostiqués étaient atteints d'hypertension depuis un certain temps déjà. L'association entre l'hypertension et des affections telles que les MCV et les AVC justifie un dépistage attentif par les fournisseurs de soins de santé, en particulier dans les populations à risque élevé. Certains sujets du groupe dont le diagnostic était récent avaient peut-être été informés par leur médecin du fait qu'ils étaient atteints d'hypertension, mais ne s'en souvenaient pas ou n'avaient pas bien compris. Toutefois, comme aucun d'entre eux ne recevait de traitement antihypertenseur, ils n'avaient probablement pas reçu de diagnostic d'hypertension avant de participer à l'étude.

Notre étude présente certaines limites. Premièrement, comme notre échantillon était composé de participants volontaires, il n'était peut-être pas représentatif de l'ensemble de la communauté ou d'autres communautés des Premières nations du Canada. En effet, une étude de dépistage reposant sur un échantillon de volontaires peut attirer principalement des personnes en bonne santé qui souhaitent en apprendre davantage sur leur état de santé, ce qui peut mener à une sous-estimation du nombre d'affections. D'un autre côté, une étude de dépistage peut attirer des personnes ayant déjà des problèmes de santé et qui souhaitent recevoir une aide médicale supplémentaire, ce qui peut mener à une surestimation de la prévalence des problèmes de santé dans une population. Nous ne pensons pas que 
notre échantillon surreprésentait l'un ou l'autre groupe, car les hommes et les femmes étaient représentés de façon égale et la répartition des âges correspondait à celle de la population admissible ${ }^{10}$. De plus, le fait que seule la moitié des membres de la communauté dont le diabète était connu a participé à l'étude constitue une autre indication de l'absence d'exagération de la prévalence des problèmes de santé dans la communauté. Aucune des quinze personnes souffrant d'insuffisance rénale terminale n'a participé à l'étude, et seuls trois des dix membres de la communauté ayant subi une amputation y ont participé ${ }^{10}$. La prévalence des affections chroniques et des facteurs de risque que nous présentons concorde sensiblement avec les recherches antérieures.

Deuxièmement, nous avons utilisé un test de mesure de la glycémie à jeun plutôt qu'un test de tolérance au glucose (glycémie à $2 \mathrm{~h}$ ). Ce dernier nous aurait peut-être permis de diagnostiquer un plus grand nombre de personnes atteintes de diabète. Notre protocole est néanmoins acceptable pour la recherche épidémiologique. Troisièmement, nous n'avons pas validé, auprès des fournisseurs de soins de santé locaux, les données relatives à l'hypertension et au diabète déclarées par les sujets. Il est donc possible que nous ayons sous-estimé la prévalence autodéclarée et, par le fait même, surestimé le nombre de cas non diagnostiqués. Cependant, nous avons déjà souligné le manque de conformité aux normes dans cette communauté en ce qui a trait à l'examen des pieds chez les personnes diabétiques ${ }^{10}$. Par conséquent, il est possible que les participants n'aient pas subi les tests pour le diabète et l'hypertension même lorsque cela était indiqué. Finalement, puisque nous avons mené une étude transversale, nous ne pouvons pas déterminer le déroulement temporel des événements.

La prévalence de l'obésité dans cette population est l'une des plus élevées ayant été signalées parmi les populations des Premières nations du Canada, en particulier chez les femmes en âge de procréer. Le nombre d'affections liées à l'obésité dans cette population est élevé, même chez les jeunes adultes, et les femmes de presque tous les groupes d'âge présentent un taux de comorbidité beaucoup plus élevé que les hommes. Il se peut qu'une proportion appréciable de participants dont l'hypertension n'était pas diagnostiquée ait été atteinte depuis un certain temps, compte tenu des associations significatives avec les autres affections chroniques et la microalbuminurie. La prévalence des facteurs de risque de maladies cardiovasculaires et rénales au sein de cette population peut laisser entrevoir une prévalence plus élevée de maladies cardiovasculaires et rénales. De plus, en raison de l'influence de l'obésité maternelle et du diabète gestationnel sur la santé des enfants, une hausse de l'obésité infantile et du diabète de type 2 pourrait survenir dans la communauté.

Toutefois, une prévalence accrue de l'obésité et des affections liées à l'obésité n'est pas inévitable. De nombreuses activités de prévention sont en cours. Premièrement, une intervention de recherche dans la communauté est axée sur la prévention du diabète gestationnel par le contrôle de la prise de poids pendant la grossesse (activité physique et alimentation). Deuxièmement, la communauté gère un centre de conditionnement physique doté d'un bon équipement et proposant des cours appropriés. Troisièmement, le centre de santé offre de la formation sur l'alimentation, l'activité physique et le mieux-être. Quatrièmement, des groupes de marches pour les jeunes et pour les adultes sont organisés par le centre de santé. Cinquièmement, des programmes d'activités sont offerts aux jeunes à l'extérieur des écoles locales. Compte tenu de l'effet bien établi de l'obésité sur la santé, une surveillance continue des affections chroniques et des facteurs de risque est néanmoins nécessaire, de même que la mise en œuvre d'autres initiatives de promotion de la santé et d'éducation sanitaire. Nous continuons de travailler, de concert avec la communauté, à la conception et à l'évaluation d'activités de prévention primaire et secondaire.

\section{Remerciements}

Nous tenons à remercier les Instituts de recherche en santé du Canada (IRSC) et le Conseil manitobain de la recherche en matière de santé d'avoir financé ce projet. Mme Riediger a reçu une bourse d'études supérieures (doctorat) des IRSC. Nous voudrions également exprimer notre reconnaissance à Mary Cheang pour son aide sur le plan de la statistique. De plus, nous remercions les membres, le personnel et les dirigeants de la communauté étudiée pour leur participation et leur engagement soutenu.

\section{Références}

1. Macaulay AC. Improving aboriginal health: how can health care professionals contribute? Can Fam Physician 2009;55(4):334-9.

2. Anand SS, Yusef S, Jacobs R, Davis AD, Yi $\mathrm{Q}$, Gerstein H, et al. Risk factors, atherosclerosis, and cardiovascular disease among Aboriginal people in Canada: the Study of Health Assessment and Risk Evaluation in Aboriginal Peoples (SHARE-AP). Lancet 2001;358(9288):1147-53.

3. Allard YE, Wilkins R, Berthelot JM. Mortalité prématurée dans les régions sociosanitaires à forte population autochtone, Rapport sur la santé 2004;15(1):55-66.

4. Lix L, Bruce S, Sarkar J, Young TK. Facteurs de risque et problèmes de santé chroniques chez les Autochtones et les non-Autochtones, Rapport sur la santé, 2009;20(4):1-10.

5. Connelly PW, Hanley AJ, Harris SB, Hegele RA, Zinman B. Relation of waist circumference and glycemic status to $\mathrm{C}$-reactive protein in the Sandy Lake Oji-Cree. Int J Obes Relat Metab Disord 2003;27(3):347-54.

6. Chateau-Degat ML, Pereg D, Egeland GM, Nieboer E, Bonnier-Viger YV, Laouan-Sidi EA, et al. Diabetes and related metabolic conditions in an aboriginal Cree community of Quebec, Canada. Canadian Journal of Diabetes 2009;33(3):156-62.

7. Oster RT, Toth EL. Differences in the prevalence of diabetes risk-factors among First Nation, Metis and non-aboriginal attending screening clinics in rural Alberta, Canada. Rural Remote Health 2009;9(2):1170. 
8. Dyck R, Osgood N, Lin TH, Gao A, Stang MR. Epidemiology of diabetes mellitus among First Nations and non-First Nations adults. CMAJ 2010;182(3):244-56.

9. Gao S, Manns BJ, Culleton BF, Tonelli M, Quan H, Crowshoe L, et al. Prevalence of chronic kidney disease and survival among aboriginal people. J Am Soc Nephrol 2007;18(11):2953-9.

10. Bruce SG, Young TK. Prevalence and risk factors for neuropathy in a Canadian First Nation community. Diabetes Care 2008;31(9):1837-41.

11. Société canadienne de physiologie de l'exercice. Guide du conseiller en condition physique et habitudes de vie (Guide du conseiller CPHV). $3^{\text {e }}$ édition. Ottawa (Ontario) (CA):SCEP, c2004.

12. Canadian Diabetes Association 2008 clinical practice guidelines for the prevention and management of diabetes in Canada. Canadian Journal of Diabetes 2008;32(Suppl 1).

13. Executive Summary of the Third Report of the National Cholesterol Education Program (NCEP) Expert Panel on Detection, Evaluation, and Treatment of High Blood Cholesterol in Adults (Adult Treatment Panel III). JAMA 2001;285(19):2486-97.

14. Armstrong DG, Lavery LA. Clinical care of the diabetic foot. Alexandria (VA): American Diabetes Association; 2005.

15. Garriguet D. L'obésité et les habitudes alimentaires de la population autochtone, Rapports sur la santé 2008;19(1):1-17.

16. Harris SB, Gittelsohn J, Hanley A, Barnie A, Wolever TM, Gao J, et al. The prevalence of NIDDM and associated risk factors in native Canadians. Diabetes Care 1997;20(2):185-7.

17. Aljohani N, Rempel BM, Ludwig S, Morris $\mathrm{M}$, Cheang $\mathrm{M}$, Murray $\mathrm{R}$, et al. Impact of diabetes on maternal-fetal outcomes in Manitoba. Relationship with ethnic and environmental factors. Clin Invest Med 2008;31(6):E338-45.
18. Dunne F, Brydon P, Smith K, Gee H. Pregnancy in women with type 2 diabetes: 12 years outcome data 1990-2002. Diabet Med 2003;20(9):734-8.

19. Young TK, Martens PJ, Taback SP, Sellers EA, Dean HJ, Cheang M, et al. Type 2 diabetes mellitus in children: prenatal and early infancy risk factors among native Canadians. Arch Pediatr Adolesc Med 2002;156(7):651-5.

20. Dyck RF, Klomp H, Tan L. From "thrifty genotype" to "hefty fetal phenotype": the relationship between high birthweight and diabetes in Saskatchewan Registered Indians. Can J Public Health 2001;92(5):340-4.

21. Masharani U, Goldfine ID, Youngren JF. Influence of gender on the relationship between insulin sensitivity, adiposity, and plasma lipids in lean nondiabetic subjects. Metabolism 2009;58(11):1602-8.

22. O’Dea K, Cunningham J, Maple-Brown L, Weeramanthri T, Shaw J, Dunbar T, et al. Diabetes and cardiovascular risk factors in urban indigenous adults: results from the DRUID study. Diabetes Res Clin Pract 2008;80(3):483-9.

23. Makaryus AN, Akhrass P, McFarlane SI. Treatment of hypertension in metabolic syndrome: implications of recent clinical trials. Curr Diab Rep 2009;9(3):229-37.

24. Amankwah E, Campbell NR, Maxwell C, Onysko J, Quan H. Why some adult Canadians do not have blood pressure measured. J Clin Hypertens (Greenwich) 2007;9(12):944-51. 